\section{Population Pharmacokinetic Modeling of a Subcutaneous Depot for GnRH Antagonist Degarelix}

\author{
Christoffer W. Tornøe, ${ }^{1,4}$ Henrik Agersø, ${ }^{1}$ \\ Henrik A. Nielsen, ${ }^{2}$ Henrik Madsen, ${ }^{2}$ and \\ E. Niclas Jonsson ${ }^{3}$
}

Received December 2, 2003; accepted January 9, 2004

\begin{abstract}
Purpose. The objective of this study is to develop a population pharmacokinetic (PK) model that describes the subcutaneous (SC) depot formation of gonadotropin-releasing hormone (GnRH) antagonist degarelix, which is being developed for treatment of prostate cancer, exhibiting dose-volume and dose-concentration dependent absorption.

Methods. The PK analysis is made in NONMEM through joint analysis of data from two phase I clinical studies; an intravenous infusion study and a single SC dose escalation study. The SC absorption is modeled using an approximation to Ficks' second law of diffusion out of a spherical depot. The dose-volume effect on the SC release is estimated using a B-spline basis whereas the bioavailability is modeled as a function of the dose-concentration.

Results. The SC depot model is approximated by using two concentric spherical compartments for the SC absorption combined with a two-compartment disposition model. The results indicate that the volume effect is most apparent at low injection volumes whereas the effect is diminishing at higher injection volumes. The doseconcentration effect on the bioavailability is estimated to decrease at increasing dose-concentrations.

Conclusions. The presented SC depot model describes the PK profile of GnRH antagonist degarelix. This modeling approach might also be applicable for other depot-formulated drugs exhibiting complex PK profiles.
\end{abstract}

KEY WORDS: degarelix; NONMEM; population pharmacokinetic modeling; prostate cancer; subcutaneous depot.

\section{INTRODUCTION}

Prostate cancer (PC) is the most common cancer among men and is responsible for almost $3 \%$ of deaths in men older than 55 years (1). The potency of gonadotropin-releasing hor-

\footnotetext{
${ }^{1}$ Clinical Pharmacology and Experimental Medicine, Ferring Pharmaceuticals A/S, Copenhagen, Denmark.

${ }^{2}$ Informatics and Mathematical Modelling, Technical University of Denmark, Lyngby, Denmark.

${ }^{3}$ Department of Pharmaceutical Biosciences, Division of Pharmacokinetics and Drug Therapy, Uppsala University, Uppsala, Sweden. ${ }^{4}$ To whom correspondence should be addressed. (e-mail: christoffer. tornoe@ferring.com)

ABBREVIATIONS: $C$, concentration $(\mathrm{ng} / \mathrm{ml}) ; C L$, clearance $(1 / \mathrm{h})$; $C V$, coefficient of variation $(\%) ; D$, diffusion constant $\left(\mathrm{cm}^{2} / \mathrm{h}\right) ; \partial$, partial derivative; $\epsilon$, residual error; $\eta$, interindividual variability; $f$, flow (ng/h); $F$, absolute bioavailability; $F R$, fraction of SC dose in outermost spherical shell; $J$, flux $\left[\mathrm{ng} /\left(\mathrm{h} \mathrm{cm}^{2}\right)\right] ; k_{a}$, absorption rate constant $\left(\mathrm{h}^{-1}\right) ; \omega^{2}$, interindividual variance; $q$, amount of drug (ng); $Q$, intercompartmental clearance $(1 / \mathrm{h}) ; r$, radial distance $(\mathrm{cm}) ; R_{d}$, depot radius $(\mathrm{cm})$; RSE, relative standard error $(\%)$; $\sigma^{2}$, residual error variance; $t$, time (h); $t_{1 / 2}$, half-life (h); $V$, volume (l).
}

mone $(\mathrm{GnRH})$ and its analogues as stimulators or inhibitors of pituitary gonadotropin secretion have made them highly useful in the therapy of sex hormone-dependent tumors. GnRH agonists have the disadvantage of producing an initial stimulatory effect on luteinizing hormone ( $\mathrm{LH})$ and folliclestimulating hormone (FSH) secretion from the pituitary, which acts on testicular Leydig cells to stimulate de novo synthesis of androgens, primarily testosterone, on which the growth of PC cells depends $(2,3)$. The continuous stimulation of the pituitary by GnRH agonists subsequently results in down-regulation of GnRH receptors followed by receptor desensitization and thereby suppression of gonadal steroids (4). The initial testosterone surge is avoided with GnRH antagonists causing immediate suppression of gonadotropin secretion and thereby resulting in medical castration by competitive blockade of the GnRH receptors at the pituitary $(5,6)$. Degarelix is a new long-acting GnRH antagonist that currently is being developed for PC treatment (7-9).

The major barrier for subcutaneous (SC) absorption into the systemic circulation is usually modeled as a first-order rate process where the movement of drug from the injection site to the blood vessels is assumed to be the rate-limiting step (10). Degarelix is administered by SC injections, which results in the formation of a spontaneous SC depot from which the drug is slowly released (9). The simplifying assumptions about first-order absorption kinetics can therefore not be applied due to the complex absorption process out of the SC depot. The rate-limiting step for SC-administered degarelix may thus not be the absorption process but instead the diffusion out of the SC depot, which seems to depend on both the volume and concentration of the injected dosing solution, that is, the doseconcentration and dose-volume. The objective of the current nonlinear mixed-effects analysis is to characterize and model the pharmacokinetics (PK) of degarelix. In order to develop successfully degarelix, it is essential to understand the factors controlling the release characteristics to be able to optimize the dosing regimens. Assuming that the SC absorption can be modeled as diffusion out of a spherical depot, the hypotheses is that the current model will be able to explain the doseconcentration and dose-volume dependent PK of degarelix and possibly other depot formulated drugs.

\section{MATERIALS AND METHODS}

\section{Compound}

Degarelix [FE200486, Ac-D-2Nal-D-4Cpa-D-3Pal-Ser4Aph (L-hydroorotyl)-D-4Aph (carbamoyl)-Leu-ILys-Pro-DAla- $\left.\mathrm{NH}_{2}\right]$ is a linear decapeptide amide containing seven unnatural amino acids (7). It is a long-acting competitive GnRH antagonist with high affinity and selectivity for GnRH receptors and showing high water solubility and low histaminereleasing properties (7). Degarelix is reconstituted in a mannitol solution immediately before injection. After SC administration, degarelix spontaneously forms a gel-like depot at high concentrations (in the $\mathrm{mg} / \mathrm{ml}$ range) when it comes into contact with body fluids. The self-depoting formation results in a sustained release of degarelix and a prolonged duration of action in terms of testosterone suppression (9). 


\section{Study Design}

Results from two phase I studies were included in the current population PK modeling analysis.

\section{Study I}

The SC study was designed as a single center, randomized, double blind, placebo-controlled, parallel-groups, doseescalation trial with single SC doses of degarelix to 80 healthy male subjects. The following dose-levels were included in the study: $0.5,2.0,5.0,10,30$, and $40 \mathrm{mg} / \mathrm{subject}$ with doseconcentrations between 5 and $30 \mathrm{mg} / \mathrm{ml}$ and dose-volumes between 0.1 and $2.0 \mathrm{ml}$ administered as either a single or two injections (Table I). Eight subjects were randomized at each group to active or placebo treatment with a ratio of $3: 1$. Blood samples were collected pre-dose and $0.08,0.25,0.5,0.75,1$, 1.5, 2, 4, 6, 8, 12, 24, 48, 96, 216, 312, 480, 696, 864, 1032, 1224, and $1416 \mathrm{~h}$ after dosing from all subjects.

\section{Study II}

The intravenous (IV) study was designed as a single center, open label, escalating study with sequential intravenous treatment groups with 24 healthy male subjects enrolled in the study. Four treatment groups received degarelix administered as an intravenous infusion with six subjects in each group receiving doses of $1.5,6,15$, and $30 \mu \mathrm{g} / \mathrm{kg}$, respectively (Table I). The two lowest dosing regimes were infused over 15 min whereas the infusion lasted $45 \mathrm{~min}$ for the two highest dosing regimens. Blood samples were collected according to the following schedule: pre-dose; during the infusion; at 5, 10, $15,30,45,60 \mathrm{~min}$; and at 2, 4, 8, 12, 24, 36, and $48 \mathrm{~h}$ after infusion stop.

\section{Bioanalytic Analysis}

Degarelix plasma concentrations were measured according to Good Laboratory Practice (GLP). Plasma samples from study I were measured by a radioimmunoassay (RIA), whereas plasma samples from study II were analyzed using liquid chromatography with tandem mass spectrometric de-

Table I. Experimental Design: Dosing Schemes for the SC and IV Studies

\begin{tabular}{cllcc}
\hline Group & Dose-level & Dose-volume & $\begin{array}{c}\text { Dose- } \\
\text { concentration }\end{array}$ & Route \\
\hline 1 & $0.5 \mathrm{mg}$ & $0.1 \mathrm{ml}$ & $5 \mathrm{mg} / \mathrm{ml}$ & $\mathrm{SC}$ \\
2 & $2.0 \mathrm{mg}$ & $0.4 \mathrm{ml}$ & $5 \mathrm{mg} / \mathrm{ml}$ & $\mathrm{SC}$ \\
3 & $5.0 \mathrm{mg}$ & $0.5 \mathrm{ml}$ & $10 \mathrm{mg} / \mathrm{ml}$ & $\mathrm{SC}$ \\
4 & $10.0 \mathrm{mg}$ & $1.0 \mathrm{ml}$ & $10 \mathrm{mg} / \mathrm{ml}$ & $\mathrm{SC}$ \\
5 & $20.0 \mathrm{mg}$ & $1.0 \mathrm{ml}$ & $20 \mathrm{mg} / \mathrm{ml}$ & $\mathrm{SC}$ \\
6 & $40.0 \mathrm{mg}$ & $1.0 \mathrm{ml} \times 2 *$ & $20 \mathrm{mg} / \mathrm{ml}$ & $\mathrm{SC}$ \\
7 & $40.0 \mathrm{mg}$ & $2.0 \mathrm{ml} \times 2 *$ & $10 \mathrm{mg} / \mathrm{ml}$ & $\mathrm{SC}$ \\
8 & $40.0 \mathrm{mg}$ & $2.0 \mathrm{ml}$ & $20 \mathrm{mg} / \mathrm{ml}$ & $\mathrm{SC}$ \\
9 & $30.0 \mathrm{mg}$ & $2.0 \mathrm{ml}$ & $15 \mathrm{mg} / \mathrm{ml}$ & $\mathrm{SC}$ \\
10 & $30.0 \mathrm{mg}$ & $1.0 \mathrm{ml}$ & $30 \mathrm{mg} / \mathrm{ml}$ & $\mathrm{SC}$ \\
$\mathrm{A}$ & $1.5 \mu \mathrm{g} / \mathrm{kg}$ & $0.3 \mathrm{ml} / \mathrm{kg}$ & $5 \mu \mathrm{g} / \mathrm{ml}$ & IV $(15 \mathrm{~min})$. \\
B & $6.0 \mu \mathrm{g} / \mathrm{kg}$ & $1.2 \mathrm{ml} / \mathrm{kg}$ & $5 \mu \mathrm{g} / \mathrm{ml}$ & IV $(15 \mathrm{~min})$. \\
C & $15.0 \mu \mathrm{g} / \mathrm{kg}$ & $3.0 \mathrm{ml} / \mathrm{kg}$ & $5 \mu \mathrm{g} / \mathrm{ml}$ & IV $(45 \mathrm{~min})$. \\
D & $30.0 \mu \mathrm{g} / \mathrm{kg}$ & $6.0 \mathrm{ml} / \mathrm{kg}$ & $5 \mu \mathrm{g} / \mathrm{ml}$ & IV (45 min.) \\
\hline
\end{tabular}

SC, subcutaneous; IV, intravenous.

* These groups received the dose as two SC injections. tection (LC-MS/MS). The lower limit of quantification (LLOQ) of the RIA was $0.1 \mathrm{ng} / \mathrm{ml}$ whereas that of the LC$\mathrm{MS} / \mathrm{MS}$ method was $0.5 \mathrm{ng} / \mathrm{ml}$. Both methods were validated according to current guidelines for bioanalytical samples.

\section{Data Analysis}

The nonlinear mixed-effects modeling was performed using NONMEM version V (11) using the first-order conditional estimation (FOCE) method. The hierarchical model structure was modeled as the following two-stage hierarchy (12).

At the first-stage model, the intraindividual (residual) variability describing the difference between the individual predicted values and the observations was modeled using an additive residual error model on the log-scale corresponding to a constant coefficient of variation $(\mathrm{CV})$ model on the untransformed scale, that is,

$$
\log y_{i j}=\log \hat{y}_{i j}+\epsilon_{i j}
$$

where $y_{i j}$ is the $j$ th observation for the $i$ th individual, $\hat{y}_{i j}$ is the corresponding model prediction, and $\epsilon_{i j}$ is the residual error term, which is independent and identically distributed (iid) with mean zero and variance $\sigma^{2}$.

At the second-stage model, the interindividual variability (IIV) model relates the parameters of the different individuals using an exponential model, that is

$$
\theta_{i}=\theta_{\text {pop }} \cdot \exp \left(\eta_{i}^{\theta}\right)
$$

where $\theta_{i}$ denotes an arbitrary PK parameter for individual $i$, $\theta_{\text {pop }}$ is the corresponding population parameter, while $\eta^{\theta}$ is a zero-mean, symmetrically distributed variable with variance $\omega_{\theta}^{2}$ to distinguish the $i$ th subject's parameter from the population mean.

Individual estimates of the PK parameters were obtained as empirical (POSTHOC) Bayes estimates. Model selection was based on goodness-of-fit (GOF) graphical analysis of residuals and predictions using Xpose 3.0 (13), distribution of residuals and weighted residuals, and on the maximum likelihood objective function value (OFV), which is approximately $\chi^{2}$-distributed. Possible covariate relationships were investigated through graphical analysis of individual parameter estimates from the model vs. covariates.

\section{Subcutaneous Depot Model}

The SC depot model relies on the principles of diffusion out of a spherical SC depot originally proposed for the absorption of SC injected insulin (14-16). Diffusion is the mechanism by which matter moves from one part of a system to another by means of random molecular (Brownian) motion. Diffusion occurs in response to a concentration gradient expressed as the change in concentration due to a change in position.

In the mid-1800s, Adolf E. Fick (17) introduced two differential equations that quantified the diffusion through thin membranes. Fick's first law of diffusion out of a sphere states that in one-dimensional steady-state diffusion, the flux $J$ is proportional to the negative concentration gradient expressed by

$$
J=-D \frac{\partial C}{\partial r}
$$


where $D$ is the diffusion constant, $C$ is the concentration in the SC depot, and $r$ is the radial distance from the core of the sphere. The negative sign indicates that $J$ is positive when movement is down the gradient.

Under non-steady state conditions, Fick's second law of diffusion is derived from an instationary differential mass balance over a spherical control volume combined with Fick's first law of diffusion, that is,

$$
\frac{\partial C}{\partial t}=D \frac{1}{r^{2}} \frac{\partial}{\partial r}\left(r^{2} \frac{\partial C}{\partial r}\right), \quad 0<r \leq R_{d}, \quad t \geq 0
$$

where $R_{d}$ is the radius of the depot and $t$ is the time since the $\mathrm{SC}$ administration. Fick's second law states that the rate of change of concentration in a volume element is proportional to the rate of change of the concentration gradient at that point in the diffusional field. The initial concentration in the sphere is equal to the the dose-concentration $C_{0}$ while the two boundary conditions for the system state that the concentration in the core of the sphere is limited and that the change in concentration at the boundary of the sphere is proportional to the amount released into the plasma.

NONMEM was originally intended for estimation of parameters in nonlinear mixed-effects models described by firstorder ordinary differential equations (ODEs). It is therefore not possible to specify partial differential equations (PDEs) in NONMEM as the one specified in Eq. (4) with time $t$ and radial distance $r$ as independent variables. As a consequence of NONMEM not being able to handle PDEs, a numerical approximation of Fick's second law of diffusion is obtained by spatial discretization of the SC depot into concentric spherical shells (16). By assuming spatially constant flow $f_{i}$ from a shell with radius $\bar{R}_{i}$ to a shell with radius $\bar{R}_{i+1}$, Fick's first law of diffusion yields

$$
J=\frac{f_{i}}{4 \pi r^{2}}=-D \frac{\partial C}{\partial r}, \quad \bar{R}_{i}<r<\bar{R}_{i+1}
$$

with

$$
\bar{R}_{i+1}=\frac{1}{\sqrt[3]{2}} \sqrt[3]{R_{i+1}^{3}+R_{i}^{3}}
$$

which is the average radius that divides the volume of spherical shell $i+1$ in two equal parts. After integration of Eq. (5) with respect to $r$ from $\bar{R}_{i}$ to $\bar{R}_{i+1}$ and $C$ from $C_{i}$ to $\mathrm{Ci}_{i+1}$, the following expression for the flow $f_{i}$ is obtained.

$$
f_{i}=4 \pi D \frac{\bar{R}_{i+1} \bar{R}_{i}}{\bar{R}_{i}-\bar{R}_{i+1}}\left(C_{i+1}-C_{i}\right)
$$

The time derivative of the SC concentration in shell $i+1$ can thereby be approximated by

$$
\frac{\partial C_{i+1}}{\partial t} \approx \frac{C_{i+1, n+1}-C_{i+1, n}}{\Delta t}=-\frac{1}{V_{i+1}}\left(f_{i+1, n}-f_{i, n}\right)
$$

where $V_{i+1}=\frac{4}{3} \pi\left(R_{i+1}^{3}-R_{i}^{3}\right)$ is the volume of shell $i+1$ and $C_{i+1}$ is the position-averaged concentration of drug in shell $i+1$.

The ODE for the SC concentration in shell $i+1$ can thereby be expressed as

$$
\begin{aligned}
\frac{d C_{i+1}}{d t}= & \frac{3 D}{R_{i+1}^{3}-R_{i}^{3}}\left[\frac{\bar{R}_{i+1} \bar{R}_{i}}{\bar{R}_{i+1}-\bar{R}_{i+1}}\left(C_{i}-C_{i+1}\right)-\frac{\bar{R}_{i+2} \bar{R}_{i+1}}{\bar{R}_{i+2}-\bar{R}_{i+1}}\right. \\
& \left.\left(C_{i+1}-C_{i+2}\right)\right]
\end{aligned}
$$

which can be implemented directly into the NONMEMNMTRAN control stream.

\section{B-Spline Basis Estimation of Effective Depot-Volume}

The semiquantitative model for the dose-volume effect on the SC release is considered next. To be able to use the SC depot model, it is necessary to specify or estimate the effective depot-volume. One approach would be to use the SC injected dose-volume as the depot-volume thereby assuming instantaneous SC depot formation of a rigid gel. Initial results indicated that this approach would not work in the current analysis due to slow depot formation, initial absorption of the vehicle, or diminished dose-volume effect at high or low injection volumes. Another possibility would be to model the effective depot-volume using a B-spline basis. By using a Bspline basis, the only assumption made about the functional relationship between the unmeasured effective depot-volume and the SC injected dose-volume is that it is a piecewise linear function.

A spline function $f(x)$ is a piecewise polynomial function defined on an interval with specified continuity constraints The mathematical definition is

$$
f(x)=\sum_{i=1}^{p} \phi_{i} B_{i}(x)
$$

where the $\phi_{i}$ values are the parameters (to be estimated), and the $B_{i}(x)$ values are the basis functions evaluated at the predictor variable $x$. The number of parameters of a B-spline basis of order 2 (linear) equals the number of breakpoints also known as internal knots plus two boundary knots (see Refs. 18 and 19 for further details).

Next, a set of linear restrictions are stated for the spline function. The spline function $f(x)$ should take the value of zero when a dose-volume of $0 \mathrm{ml}$ is injected (i.e., no intercept). This restriction $[f(0)=0]$ leads to the reduction of one parameter, that is,

$$
f(x)=\sum_{i \neq 1}^{p} \phi_{i}\left[B_{i}(x)-\frac{B_{i}(0)}{B_{1}(0)} B_{1}(x)\right]=\sum_{i=2}^{p} \phi_{i} \beta_{i}(x)
$$

By further restricting a unity slope from the boundary knot at $0 \mathrm{ml}$, that is,

$$
f^{\prime}\left(0^{+}\right)=\sum_{i \neq 1}^{p} \phi_{i} \beta_{i}^{\prime}(0)=1
$$

the spline function now consists of $p-2$ basis functions and can be expressed by

$f(x)=\frac{\beta_{2}(x)}{\beta_{2}^{\prime}(0)}+\sum_{i \neq 1,2}^{p} \phi_{i}\left[\beta_{i}(x)-\frac{\beta_{i}^{\prime}(0)}{B_{2}^{\prime}(0)} \beta_{2}(x)\right]$

The restriction in Eq. (12) is made to make the effective depot-volume estimate at the different dose-volumes relative to the lowest dose-volume of $0.1 \mathrm{ml}$. 
In order to separate the dose-concentration and dosevolume effect on the SC release, it is furthermore necessary to impose monotonicity for the spline function, that is, the effective depot-volume should increase at increasing dosevolumes $\left[\forall x: f^{\prime}(x)>0\right]$. To satisfy the set of inequality constraints

$$
\phi_{i} \leq \phi_{i+1} \text { for } i=1, \ldots, p-1
$$

the spline coefficients are reparameterized according to Refs. 20 and 21 as

$$
\phi_{i}=\sum_{j=1}^{i} \psi_{j}, \text { for } i=1, \ldots, p
$$

where $\psi_{i}$ is the increment between the knots $\varphi_{i}$ and $\varphi_{i-1}$ restricted to be positive.

The inequality constraints in Eq. (14) along with the reparameterization in Eq. (15) are adequate restrictions for obtaining a monotone nondecreasing spline function (22).

\section{RESULTS}

The absolute bioavailability of SC-administered degarelix in healthy male volunteers is determined through joint analysis of both IV and SC data from two parallel clinical studies using a population PK modeling approach.

The PK of IV-administered degarelix was best described by a two-compartment disposition model with first-order elimination from the central compartment. Administration of SC doses of degarelix demonstrated flip-flop pharmacokinetics, that is, the absorption process is the rate-limiting step. In order to describe the absorption flip-flop phenomenon, a SC absorption model with two absorption compartments representing fast and slow SC release, as proposed in Ref. 23, was initially tested. This model could account for the initial fast SC release followed by prolonged release from a SC depot. In order to improve the model's ability to quantify and predict the different PK profiles at different dose-concentrations and dose-volumes, a SC depot model with diffusion out of concentric spherical compartments was investigated. The combined structural PK model for degarelix is illustrated in Fig. 1. It is obtained by combining the SC depot model in Eq. (9)

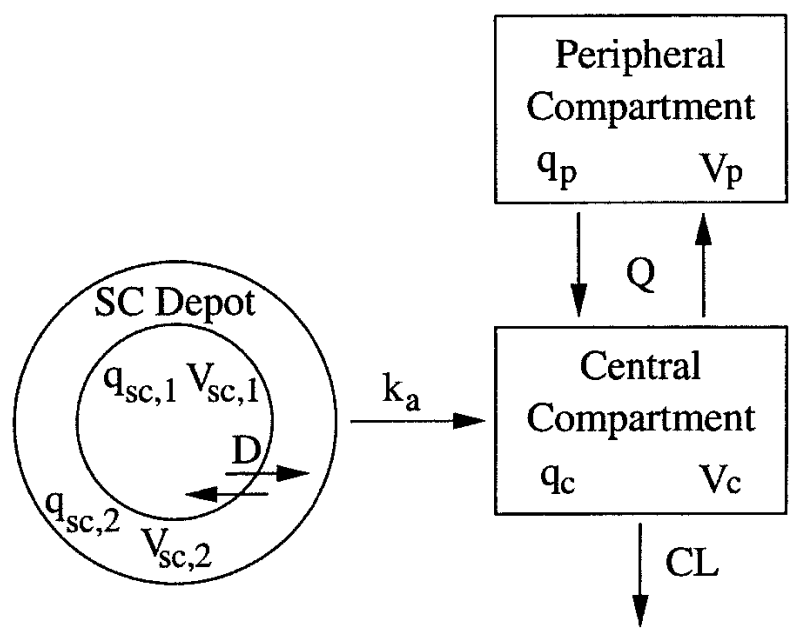

Fig. 1. Two-compartment disposition model with diffusion out of a SC depot modeled as two concentric spherical compartments. The PK parameters are explained in the text. using two concentric spherical shells with a two-compartment disposition model. The differential equations governing the model are

$$
\begin{aligned}
\frac{d q_{s c, 1}}{d t} & =-\frac{3 D V_{s c, 1}}{R_{1}^{3}} \frac{\bar{R}_{2} \bar{R}_{1}}{\bar{R}_{2}-\bar{R}_{1}}\left(\frac{q_{s c, 1}}{V_{s c, 1}}-\frac{q_{s c, 2}}{V_{s c, 2}}\right) \\
\frac{d q_{s c, 2}}{d t} & =\frac{3 D V_{s c, 2}}{R_{2}^{3}-R_{1}^{3}} \frac{\bar{R}_{2} \bar{R}_{1}}{\bar{R}_{2}-\bar{R}_{1}}\left(\frac{q_{s c, 1}}{V_{s c, 1}}-\frac{q_{s c, 2}}{V_{s c, 2}}\right)-k_{a} F q_{s c, 2} \\
\frac{d q_{c}}{d t} & =k_{a} F q_{s c, 2}+Q\left(\frac{q_{p}}{V_{p}}-\frac{q_{c}}{V_{c}}\right)-C L \frac{q_{c}}{V_{c}} \\
\frac{d q_{p}}{d t} & =-Q\left(\frac{q_{p}}{V_{p}}-\frac{q_{c}}{V_{c}}\right)
\end{aligned}
$$

where $q_{s c, 1}, q_{s c, 2}, q_{c}$, and $q_{p}$ are the state variables for the amount of drug in the two SC compartments and the central and peripheral compartments, respectively, while $V_{s c, 1}, V_{s c, 2}$, $V_{c}$, and $V_{p}$ are the corresponding volumes. $D$ is the diffusion constant, $k_{a}$ is the first-order rate constant for the absorption, $F$ is the absolute bioavailability, $Q$ is the intercompartmental clearance, and $C L$ is the clearance.

The movement of drug between the spherical SC shells is controlled by the diffusion constant $D$. The drug is cleared from the outermost spherical shell of the SC depot through the SC tissue and into the systemic circulation by the firstorder rate constant $k_{a}$ as the movement of drug is thought to be perfusion rate-limited in the SC tissue.

The fraction of the SC dose going into the outermost spherical shell $F R$ is estimated to account for the initial fast fraction cleared by the first-order absorption constant $k_{a}$ before the depot formation. The fraction parameter $F R$ determines the fraction of the initial amount of drug in the two spherical shells along with the radius and volume of the subcutaneous compartments. Because the fraction $F R$ can only assume values between 0 and 1, it is necessary to constrain $F R$ by logit-transformation, that is,

$$
\begin{aligned}
\kappa & =\ln \frac{F R}{1-F R} \\
F R_{i} & =\frac{\exp \left(\kappa+\eta_{i}^{F R}\right)}{1+\exp \left(\kappa+\eta_{i}^{F R}\right)}
\end{aligned}
$$

The IIV model in Eq. (2) was applied to the parameters $k_{a}, V_{c}, V_{p}, C L, F R$, and $F$ whereas the remaining model parameters were estimated as fixed-effect parameters due to insignificant interindividual variance estimates. No covariates were found to be significant using graphical analysis and were therefore not included in the final model. The final PK parameter estimates for the SC depot model are summarized in Table II.

The primary population parameters describing the PK of degarelix include the clearance and intercompartmental clearance, which are estimated to $3.32 \mathrm{l} / \mathrm{h}$ and $5.56 \mathrm{l} / \mathrm{h}$, respectively. The estimate of the plasma volume is 8.881 whereas that of the peripheral volume is $40.9 \mathrm{l}$, which results in an estimate of 49.81 for the total volume of distribution. The absorption half-life $t_{1 / 2, a b s}$ is $32.9 \mathrm{~h}$, and the diffusion constant $D$ is estimated to $6.03 \times 10^{-6} \mathrm{~cm}^{2} / \mathrm{h}$ and is thereby the controlling factor for the $\mathrm{SC}$ release. The fraction going into the outer spherical compartment accounting for the initial fast SC 
Table II. Summary of Population PK Parameters and Variance Estimates for Degarelix Following IV and SC Administration

\begin{tabular}{|c|c|c|c|c|c|}
\hline \multirow[b]{2}{*}{ Parameter } & \multirow[b]{2}{*}{ Units } & \multicolumn{2}{|c|}{ Population mean } & \multicolumn{2}{|c|}{ IIV } \\
\hline & & Value & $\begin{array}{l}\text { RSE } \\
(\%)\end{array}$ & $\begin{array}{l}\text { CV } \\
(\%)\end{array}$ & $\begin{array}{l}\text { RSE } \\
(\%)\end{array}$ \\
\hline$C L$ & $1 / h$ & 3.32 & 4 & 16 & 13 \\
\hline$V_{c}$ & 1 & 8.88 & 7 & 51 & 22 \\
\hline$V_{p}$ & 1 & 40.9 & 7 & 29 & 31 \\
\hline$Q$ & $1 / h$ & $5.56 *$ & 6 & - & - \\
\hline$k_{a}$ & $1 / \mathrm{h}$ & 0.0211 & 8 & 36 & 10 \\
\hline$D$ & $\mathrm{~cm}^{2} / \mathrm{h}$ & $6.03 \times 10^{-6 *}$ & 11 & - & - \\
\hline$F R$ & & 0.147 & 5 & 3 & 17 \\
\hline$\psi_{0.45}$ & $\mathrm{ml}$ & $0.0^{*}, \dagger$ & - & - & - \\
\hline$\psi_{1.0}$ & $\mathrm{ml}$ & $0.0682^{*}$ & 49 & - & - \\
\hline$\psi_{2.0}$ & $\mathrm{ml}$ & $0.0^{*}, \dagger$ & - & - & - \\
\hline$F_{5}$ & & $1.63 \ddagger$ & 14 & 17 & 22 \\
\hline$F_{10}$ & & $0.712 \ddagger$ & 7 & 17 & 22 \\
\hline$F_{15}$ & & $0.594 \ddagger$ & 7 & 17 & 22 \\
\hline$F_{20}$ & & $0.508 \ddagger$ & 8 & 17 & 22 \\
\hline$F_{30}$ & & $0.362 \ddagger$ & 14 & 17 & 22 \\
\hline$\sigma$ & $\%$ & 17.8 & 4 & - & - \\
\hline
\end{tabular}

PK, pharmacokinetic; IV, intravenous; SC, subcutaneous; IIV, interindividual variability; RSE, relative standard error; $\mathrm{CV}$, coefficient of variation.

* Parameters were estimated without IIV.

$\dagger$ Fixed at estimated value in order to obtain standard error estimates.

$\ddagger$ The IIV was the same for all dose-concentration dependent $F$ values.

release before the formation of a rigid gel in the SC depot is estimated to $14.7 \%$ of the total depot-volume.

The concentration-time profile of the observed, individual predicted, and population predicted plasma degarelix concentrations for the IV and SC study are shown in Fig. 2. The basic GOF graphs in Fig. 3 indicate good agreement between the observed plasma concentrations and the model predictions, as the circles are nicely scattered around the line of identity. The estimated model's ability to replicate the median concentration-time curves of the observed data was furthermore tested by simulating 100 data sets. The results did not indicate any bias between the observations and simulations (not shown). Finally, the model was validated by inspection of the residual plots discussed in Ref. 24 (e.g., WRES vs. time, WRES vs. PRED, IWRES vs. IPRED, and IWRES vs. time), which did not indicate any model misspecifications.

\section{SC Depot Concentration}

In order to implement the model in NONMEM, the numerical approximation of Fick's second law of diffusion described in Eq. (9) was used. Several different spatial discretization schemes of the SC depot have been tested with different numbers of concentric spherical shells ranging from 2 to 10 shells with equal volume or radius. Because no measurements of the SC drug concentration are available, the simplest scheme with two concentric spherical shells was chosen and found adequate at representing the SC depot. No improvements of the model were observed when using more spherical shells.

The analytical solution to the SC concentration as a function of the position in the sphere and the time since the SC injection is derived in the Appendix and is shown in Eq. (22), that is,

$$
C(r, t)=C_{0} \sum_{n=1}^{\infty} E_{n} \exp \left(-k_{n}^{2} \frac{D}{R_{d}^{2}} t\right) \frac{R_{d}}{r} \sin \left(k_{n} \frac{r}{R_{d}}\right)
$$

where $C_{0}$ is the dose-concentration. The SC concentration $C(r, t)$ is calculated using the first 50 eigenvalues $k_{\mathrm{n}}$ and expansion coefficients $E_{\mathrm{n}}$ using the uniroot and integrate functions in S-PLUS (version 6.0, Insightful Corp., Seattle, WA, USA).

By means of the analytical solution in Eq. (22), the error made in the numerical approximation of the SC depot model using two concentric spherical compartments can be assessed. In Fig. 4, the analytical solution to the SC depot concentration for group 1 in Table I is compared with the spatial approximation at three different time points, that is, 20, 43, and 59 days after administration of the SC dose. The SC depot concentration is initially equal to the dose-concentration of 5 $\mathrm{mg} / \mathrm{ml}$. After 20 days, the concentration is reduced to $0 \mathrm{mg} / \mathrm{ml}$ close to the depot boundary at $r=0.28 \mathrm{~cm}$, whereas the concentration after 59 days still is around $3 \mathrm{mg} / \mathrm{ml}$ at the core of the depot. The two solutions are further compared by plotting the exchange rate between the boundary of the SC depot and the central compartment (Fig. 5). The analytical and approximative exchange rates are calculated by $\left.k_{a} C_{s c}\right|_{r=R_{d}}$ and $k_{a} q_{s c, 2} / V_{s c, 2}$, respectively. The approximative exchange rate is higher than the analytical as the concentration in the outer spherical shell is an average concentration covering $14.7 \%$ of the total depot volume whereas the analytical concentration can be calculated at the exact depot boundary. On that basis, the spatial discretization of the SC depot yields sufficiently accurate results because the error made seems to be at an acceptable level when comparing the analytical and approximative curves in Figs. 4 and 5.

\section{Controlling Factors for the SC Depot}

The B-spline basis function for the spline transformation of the injected dose-volume to the effective depot-volume is constructed with five knots, that is, $\mathrm{p}=5$. The boundary knots are positioned at 0.0 and $2.0 \mathrm{ml}$ and the three internal knots are located at $0.1,0.45$, and 1.0 corresponding to the dose-volumes shown in Table I except for the dose-volumes of 0.4 and $0.5 \mathrm{ml}$, which have been combined to a single knot at $0.45 \mathrm{ml}$.

The B-spline basis function $f(x)$ in Eq. (13) is used in the estimation of the effective depot-volume and generated using the bs function in S-PLUS, that is,

$$
f(x)=\left[\begin{array}{l}
0.000 \\
0.100 \\
0.014 \\
0.000 \\
0.000 \\
0.000
\end{array}\right]+\left[\begin{array}{lll}
0.000 & 0.000 & 0.000 \\
0.000 & 0.000 & 0.000 \\
0.857 & 0.000 & 0.000 \\
0.909 & 0.091 & 0.000 \\
0.000 & 1.000 & 0.000 \\
0.000 & 0.000 & 1.000
\end{array}\right]\left[\begin{array}{l}
\phi_{0.45} \\
\phi_{1.0} \\
\phi_{2.0}
\end{array}\right]
$$

where each row in Eq. (23) corresponds to the effective depot-volume predictors at SC injected dose-volumes of $0.0,0.1$, $0.4,0.5,1.0$, and $2.0 \mathrm{ml}$.

The effective depot-volume estimates are relative to a depot-volume of $100 \%$ at dose-volumes of $0.1 \mathrm{ml}$. It was further necessary to constrain the B-spline basis to be monotone 

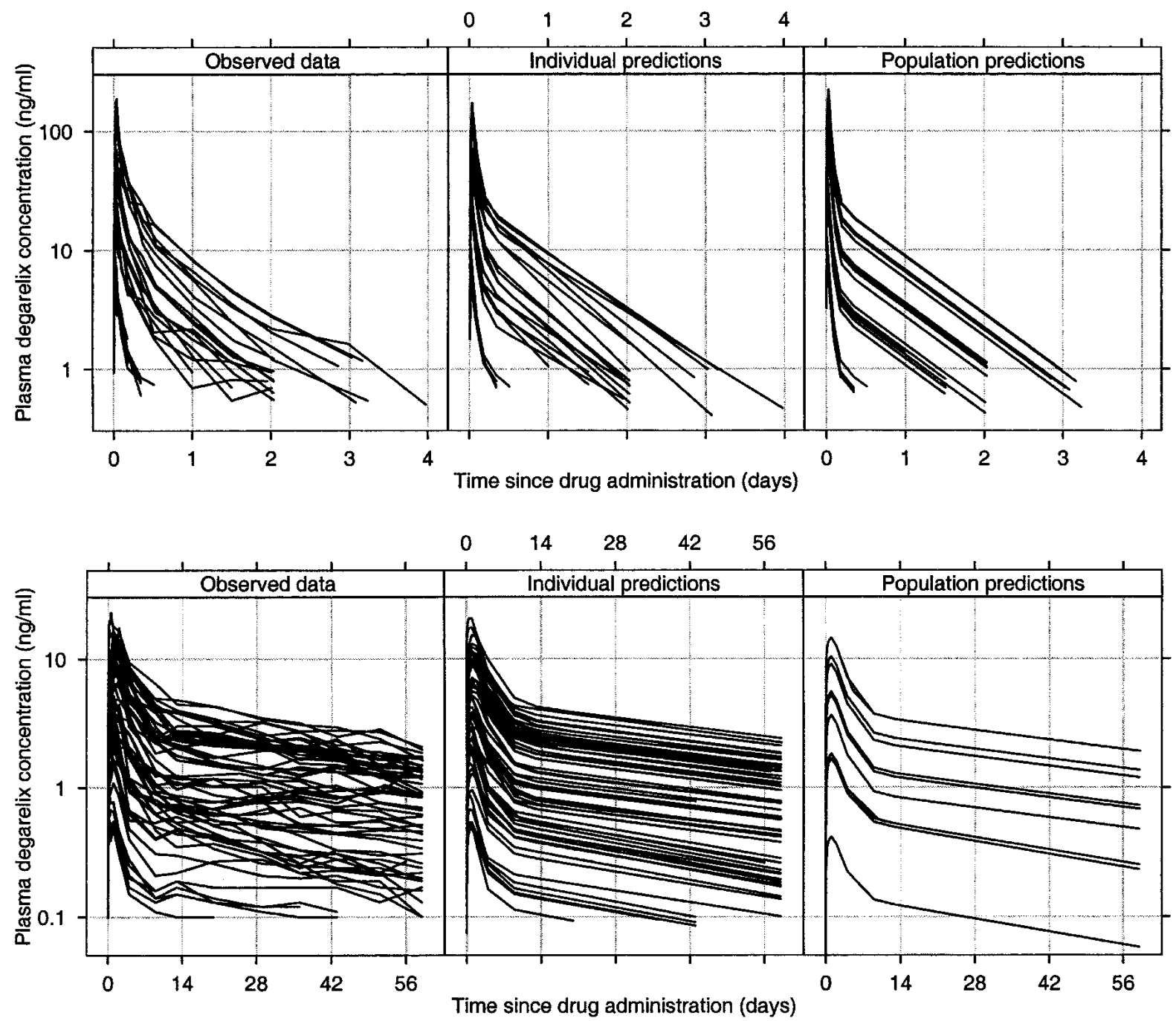

Fig. 2. Observed and predicted degarelix plasma concentration plotted on a semi-logarithmic scale with each line representing data from one individual. Left, observed data; middle, individual predictions; and right, population predictions from the IV (top) and SC (bottom) study.

nondecreasing in order to separate the dose-volume and dose-concentration effect on the SC release. The effective depot-volume estimates for dose-volumes of 0.4 and $0.5 \mathrm{ml}$ are $100 \%$ and $106 \%$, respectively. The maximum effective depot-volume is estimated to $168 \%$ at dose-volumes of $1.0 \mathrm{ml}$ and above, thereby indicating diminishing dose-volume effects between dose-volumes of 1.0 and $2.0 \mathrm{ml}$. The effective depot-volume estimates are plotted in Fig. 6 as a function of the injected dose-volume.

The bioavailability was initially attempted modeled as a function of the dose-concentration using a sigmoidal $E_{\text {max }}$ model as described in Ref. 25 , but it was not possible to estimate the model parameters. Instead, the absolute bioavailability for the five different dose-concentration groups are modeled with a fixed-effect parameter for each group and with the same random-effect parameter $\eta_{F}$. The estimated absolute bioavailability decreases at increasing doseconcentrations (Fig. 7). At the lowest dose-concentration of 5 $\mathrm{mg} / \mathrm{ml}$, the population estimate of the absolute bioavailability is $163 \%$, which is in agreement with noncompartmental analysis (NCA) (unpublished work, Ferring Pharmaceuticals
A/S). The absolute bioavailability estimate at the highest dose-level of $30 \mathrm{mg} / \mathrm{ml}$ drops to about $36 \%$, which is consistent with the results from the NCA where the estimated bioavailability is around $35-40 \%$.

\section{DISCUSSION}

The main objective of the current study was to understand the controlling factors affecting the subcutaneous depot release of GnRH antagonist degarelix. A population PK model has been developed to describe the PK concentrationtime profile following IV and SC administration. The model relies on the principles of diffusion out of a SC spherical depot with the hypotheses that the dose-volume and doseconcentration are the controlling factors affecting the SC depot release.

The complete diffusion model for a SC depot is described by a partial differential equation with both time and radial distance as independent variables. This setup is too complicated for the current population PK analysis because it is not possible with the current nonlinear mixed-effects modeling software to estimate parameters in such models. The pres- 

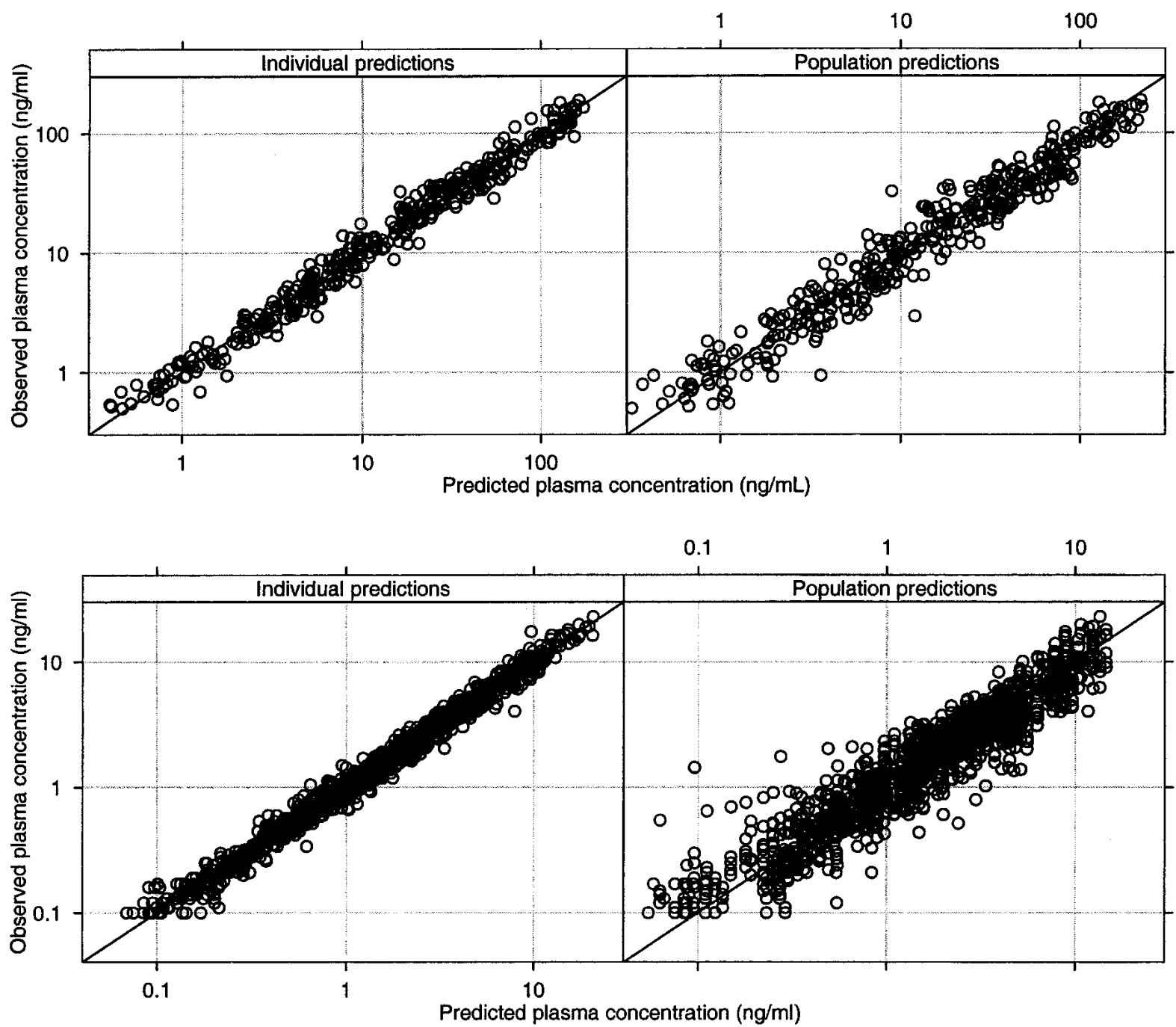

Fig. 3. Basic goodness-of-fit graphs. Plot of observed vs. individual predicted (left) and population predicted (right) degarelix plasma concentrations from the IV (top) and SC (bottom) study on a double-logarithmic scale. The solid lines are the lines of identity.

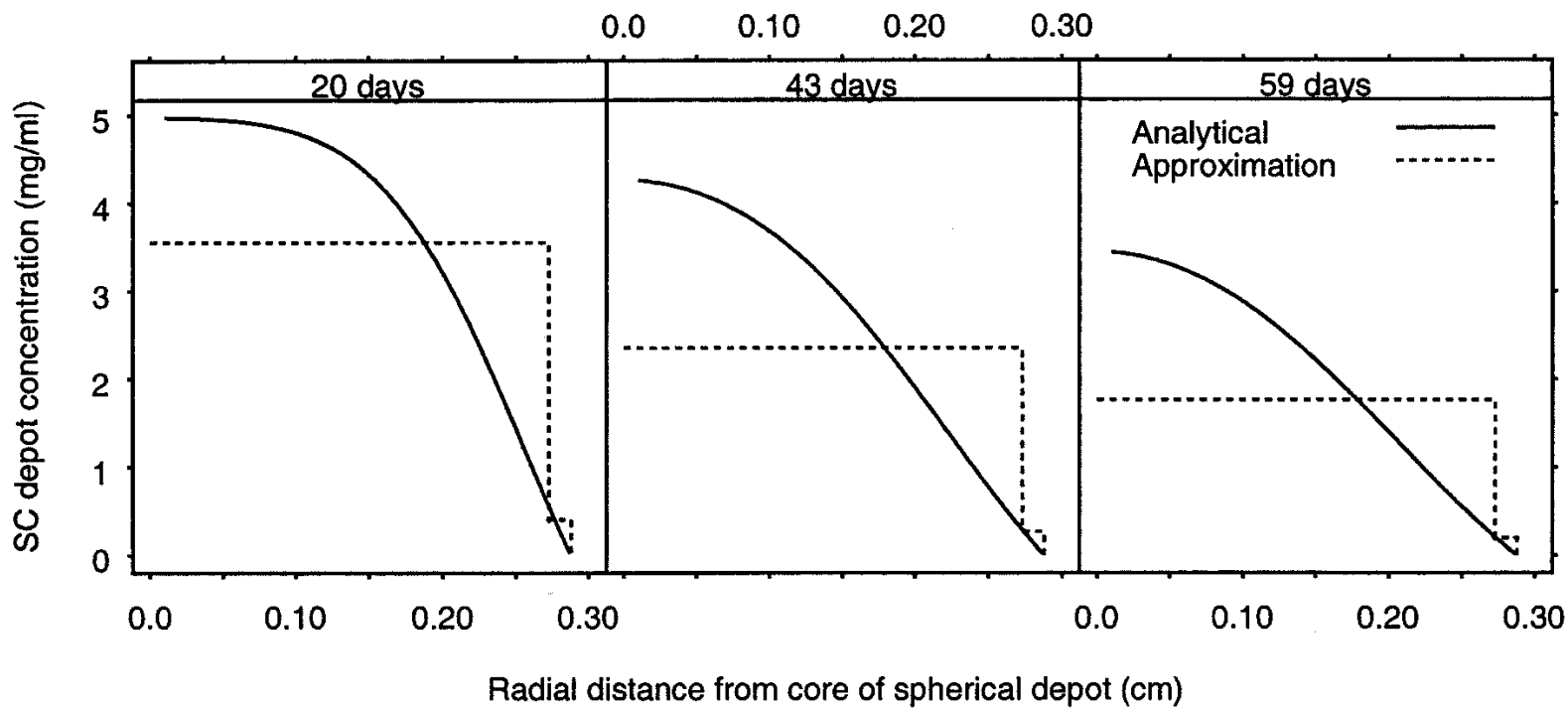

Fig. 4. SC depot concentration as a function of radial distance from the core of the spherical depot. Analytical and spatial approximation of the SC depot at times 20 (left), 43 (middle), and 59 days (right) after dose administration for group 1 (0.1 ml, $5 \mathrm{mg} / \mathrm{ml})$. 


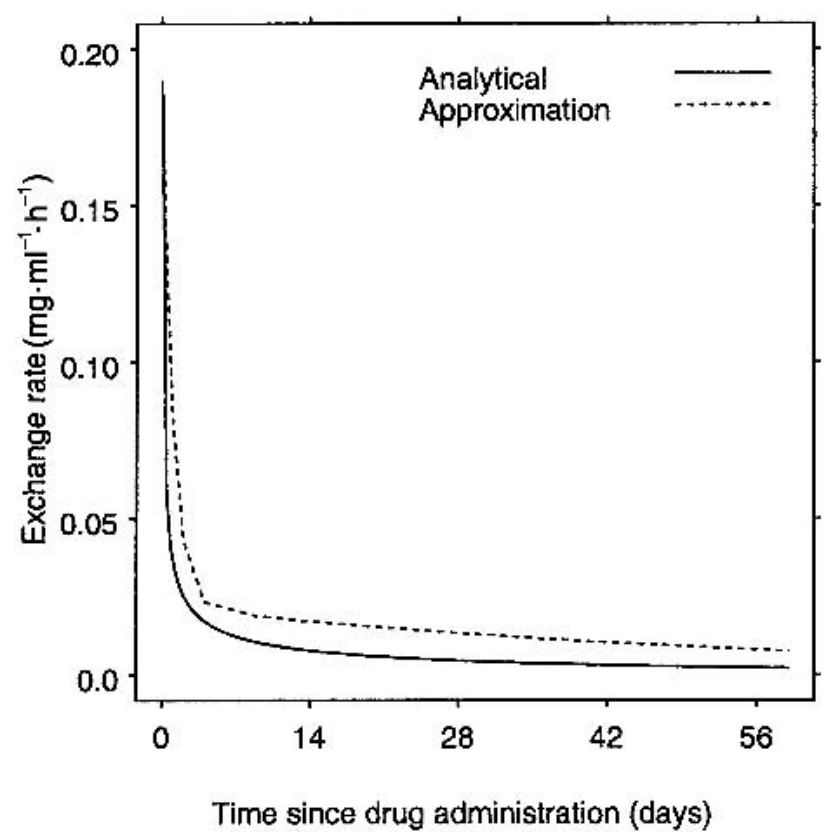

Fig. 5. Analytical solution and spatial approximation of the exchange rate between the SC depot and plasma as a function of time since drug administration.

ented discretized approximation of the SC depot yields sufficiently accurate results when comparing it with the analytical solution. The spatial discretization using only two concentric spherical compartments for the SC depot is a crude simplification of the true system, but initial results indicated no model improvements with an increasing number of shells. Further expansion of the SC depot model is not possible without additional a priori knowledge about the drug characteristics or from SC administration of labeled degarelix to obtain measurements of the SC concentration. The pharmacological relevance of the estimated diffusion constant $(D=6.03 \times$ $10^{-6} \mathrm{~cm}^{2} / \mathrm{h}$ ) is therefore perhaps not that high, since a very crude approximation of the depot is used.

The presented SC depot model provides a general approach for understanding the controlling factors affecting drug absorption. In the current analysis, the dose-volume and dose-concentration influence the SC release. Small injection volumes seem to increase the $\mathrm{SC}$ release due to shorter distance out of the SC depot and thereby faster absorption into the systemic circulation. This dose-volume effect is modeled by a B-spline basis where the functional relationship between the unmeasured effective depot-volume and the SC injected dose-volume is modeled by a piecewise linear function. The maximum effective depot-volume at dose-volumes of $1.0 \mathrm{ml}$ and above is estimated to be 1.68 times the size of the depot at dose-volumes of $0.1 \mathrm{ml}$. The explanation for observing diminishing dose-volume effects at large injection-volumes might be due to the way the SC depot is formed. After its formation, the depot undergoes a maturation stage where the density of the gel increases and the release rate decreases. At large dose-volumes, the maturation takes longer time at a given concentration resulting in easier diffusion out of the depot until the formation of a rigid gel.

The experimental design of the dose escalation SC study was originally not designed for determining a dose-volume

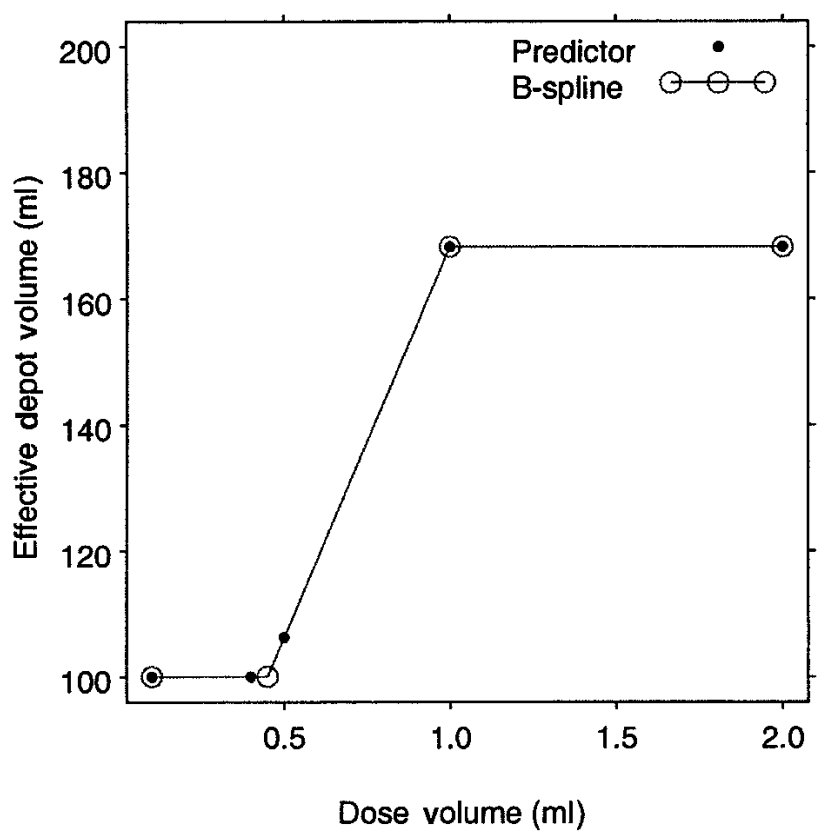

Fig. 6. Plot of the effective-depot volume estimate as a function of the injected dose-volume. The estimates are relative to a depotvolume of $100 \%$ at dose-volumes of $0.1 \mathrm{ml}$. The closed circles (-) symbolize the predictors whereas the open circles $(\bigcirc)$ represent the B-spline basis.

effect, and the design is therefore not balanced at the low dose-volumes. The effective depot-volume estimates confirm the NCA indications of a dose-volume effect, but further investigations must be performed before one can draw definitive conclusions about the effect on the SC release.

The other controlling factor for the SC absorption of degarelix is the dose-concentration. The bioavailability is ob-

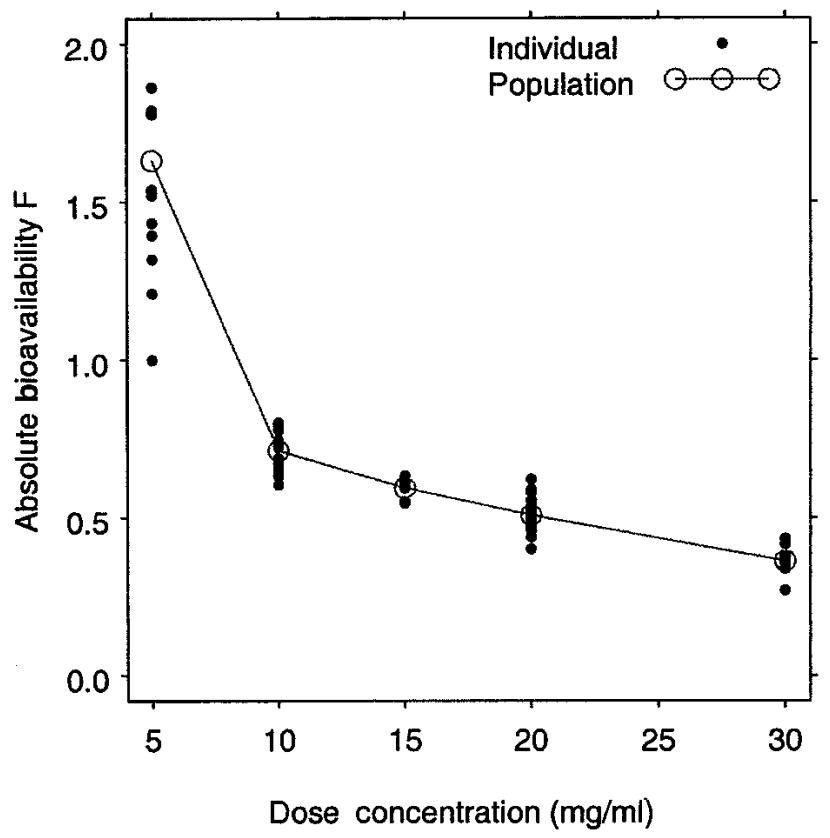

Fig. 7. Plot of the estimate of the absolute bioavailability $F$ as a function of the dose-concentration. The closed circles $(\bullet)$ symbolize the individual estimates of the absolute bioavailability whereas the open circles $(\bigcirc)$ represent the population parameters. 
served to decrease at increasing dose-concentrations. This might be due to the formation of a more rigid gel in the SC depot at high dose-concentrations resulting in increasing amounts of drug being degraded in the depot because the diffusion out of the depot is reduced. The estimate of an absolute bioavailability above $100 \%$ at the lowest doseconcentration of $5 \mathrm{mg} / \mathrm{ml}$ might be a result of high subject variation (parallel study design), uncertainty about exact dose received, and due to different analytic methods used in the IV and SC study.

Other factors that could affect the SC depot formation are the injection depth and velocity, temperature, $\mathrm{pH}$, milieu composition, along with the time from reconstitution of degarelix in the mannitol solution to the actual SC administration, but these factors are not considered in the current analysis because the information is not available.

The conclusion to draw from the analysis of the controlling factors for the SC absorption is that the choice of dosevolume would be above $1.0 \mathrm{ml}$ in order to eliminate the variability in the SC release. With respect to the doseconcentration, preclinical data suggest that the decreased bioavailability at increasing dose-concentrations levels out at concentrations above $30 \mathrm{mg} / \mathrm{ml}$, but this has yet to be confirmed in the clinic. The optimal dosing regimen in the light of the current population PK analysis would therefore be to use a dose-volume above $1.0 \mathrm{ml}$ and to adjust the doseconcentration accordingly in order to obtain the needed exposure of degarelix to suppress the testosterone concentration below castrate levels.

In summary, the population PK analysis of degarelix presents a new way of modeling a SC depot. We have presented a mechanistic PK modeling approach to understand the controlling factors affecting a SC depot and thereby a tool for optimizing the dosing regimens. The dose-volume effect on the SC release is modeled by estimating the effective depot-volume using a B-spline basis while the doseconcentration influences the bioavailability. The applicability of the presented model may be extended to other depot formulated drugs. Furthermore, the principles of diffusion can also be applied to drug absorption following oral administration, where factors such as drug solubility, permeability, and dissolution affect the drug absorption.

\section{ACKNOWLEDGMENTS}

This work was sponsored by Ferring Pharmaceuticals A/S, Denmark, the Swedish Foundation for Strategic Research, Sweden, and Center for Information Technology, Denmark.

\section{APPENDIX: ANALYTICAL SOLUTION TO SC DEPOT MODEL}

The solution to the partial differential equation (4) is found using the theory for Sturm-Liouville problems (Ref. 26).

The independent variables $r$ and $t$ in Eq. (4) are substituted with the dimensionless independent variables $x=r / R_{d}$ and $x=D / R_{d}{ }^{2} t$. The dependent variable $C(r, t)$ is made dimensionless by dividing with the injected dose-concentration
$C_{0}$, that is, $\Theta(r, t)=C(r, t) / C_{0}$. These variable substitutions reduce Eq. (4) by use of the chain rule to the following dimensionless PDE for SC diffusion.

$$
\frac{\partial \Theta}{\partial \tau}=\frac{1}{x^{2}} \frac{\partial}{\partial x}\left(x^{2} \frac{\partial \Theta}{\partial x}\right)
$$

In order to solve the standard Sturm-Liouville problem in Eq. (A1), one initial condition (IC) and two boundary conditions (BC) must be specified, that is,

$$
\begin{gathered}
\Theta(x, 0)=1 \\
\Theta(x, \tau) \text { is limited for } x \rightarrow 0^{+} \\
\left.\frac{D C_{0}}{R_{d}} \frac{\partial \Theta}{\partial x}\right|_{x=1}-\left.k_{a} C_{0} \Theta\right|_{x=1}=0, \tau \geq 0
\end{gathered}
$$

where the initial concentration in the sphere is considered equal to $C_{0}$ in Eq. (A2). The first BC in Eq. (A3) states that the concentration in the center of the sphere is limited while the second BC in Eq. (A4) states that the change in the concentration at the boundary of the sphere is assumed proportional to the amount released into the plasma.

\section{Separation of Variables}

The transformed SC concentration $\Theta(x, \tau)$ can be written as a product solution, that is,

$$
\Theta(x, \tau)=X(x) T(\tau)
$$

where $X$ and $T$ only are functions of $x$ and $\tau$, respectively.

Equation (A1) can thereby be written as

$$
x^{2} X T^{\prime}=T \frac{d}{d x}\left(x^{2} X^{\prime}\right)
$$

Because $X$ only depends on $x$ and $T$ only depends on $\tau$, and $x$ and $\tau$ are independent variables, each side of the equal sign in Eq. (A6) must be equal to a constant (i.e., $-\lambda$ ). By dividing with $T, X$, and $x^{2}$, the problem is reduced to solving the following two equations.

$$
\begin{aligned}
\frac{d}{d x}\left(x^{2} X^{\prime}\right)+\lambda x^{2} X & =0 \\
T^{\prime}+\lambda T & =0
\end{aligned}
$$

The solution to Eq. (A7) satisfying the BC in Eq. (A3) can thereby be written as

$$
\left.\left.X(x)=A \frac{1}{x} \sin (\sqrt{\lambda} x), \quad \lambda>0, \quad x \in\right] 0,1\right]
$$

The BC in Eq. (A4) leads to the following transcendent equation.

$$
\tan (k)=\frac{k}{\left(1+\frac{k_{a} R_{d}}{D}\right)}
$$

The eigenvalues are therefore those values $\lambda=k^{2}$ for which $k$ is a solution different from zero in the transcendent equation (A10), that is,

$$
\lambda_{n}=k_{n}^{2}, n=1,2, \ldots
$$


where $k_{n}$ are the positive roots in the transcendent equation (A10). The eigenfunctions $X_{n}(x)$ corresponding to the eigenvalues $\lambda_{n}$ are therefore

$$
X_{n}(x)=A_{n_{x}} \frac{1}{x} \sin \left(k_{n} x\right)
$$

Because $\lambda_{n}=k_{n}{ }^{2}$, the complete solution to Eq. (A8) for $T_{n}$ is

$$
T_{n}(\tau)=B_{n} \exp \left(-k_{n}{ }^{2} \tau\right)
$$

\section{Eigenfunction Expansion}

The dimensionless SC concentration $\Theta(x, \tau)$ can then be written as the following eigenfunction expansion

$$
\begin{aligned}
\Theta(x, \tau) & =\sum_{n=1}^{\infty} X_{n}(x) T_{n}(\tau) \\
& =\sum_{n=1}^{\infty} E_{n} \exp \left(-k_{n}^{2} \tau\right) \frac{1}{x} \sin \left(k_{n} x\right)
\end{aligned}
$$

where the expansion coefficient $E_{n}=A_{n} B_{n}$.

$E_{n}$ is determined by the IC in Eq. (A2), that is,

$$
\Theta(x, 0)=\sum_{n=1}^{\infty} E_{n} \exp \left(-k_{n}^{2} 0\right) \frac{1}{x} \sin \left(k_{n} x\right)=1
$$

The coefficients $E_{n}$ are determined by multiplication with an arbitrary eigenfunction $1 /_{x} \sin \left(k_{m} x\right)$ and by integration over the definition interval for $x$, that is,

$$
\sum_{n=1}^{\infty} E_{n} \int_{0}^{1} \frac{1}{x} \sin \left(k_{n} x\right) \frac{1}{x} \sin \left(k_{m} x\right) d x=\int_{0}^{1} \frac{1}{x} \sin \left(k_{m} x\right) d x
$$

By using the orthogonal properties for eigenfunctions, the only contribution is when $n=m$.

$$
\begin{aligned}
\int_{0}^{1} \frac{1}{x} \sin \left(k_{n} x\right) & \frac{1}{x} \sin \left(k_{m} x\right) d x= \\
& \left\{\begin{array}{rr}
0 & \text { for } n \neq m \\
\int_{0}^{1} \frac{1}{x^{2}} \sin ^{2}\left(k_{m} x\right) d x & \text { for } n=m
\end{array}\right.
\end{aligned}
$$

Equation (A17) can thereby be written as

$$
\begin{aligned}
E_{n}= & \frac{\int_{0}^{1} \frac{1}{x} \sin \left(k_{n} x\right) d x}{\int_{0}^{1} \frac{1}{x^{2}} \sin ^{2}\left(k_{n} x\right) d x}=\frac{\left[\frac{\sin \left(k_{n} x\right)}{k_{n}^{2}}-\frac{x \cos \left(k_{n} x\right)}{k_{n}}\right]_{0}^{1}}{\left[\frac{x}{2}-\frac{\sin \left(k_{n} x\right) \cos \left(k_{n} x\right)}{2 k_{n}}\right]_{0}^{1}} \\
= & \frac{\frac{\sin \left(k_{n}\right)}{k_{n}^{2}}-\frac{\cos \left(k_{n}\right)}{k_{n}}}{\frac{1}{2}-\frac{\sin \left(k_{n}\right) \cos \left(k_{n}\right)}{2 k_{n}}}
\end{aligned}
$$

The SC concentration $C(r, t)$ can be found by substituting the dimensionless variables in Eq. (A15) with $r$, $t$, and $C$ thereby obtaining the analytical solution to Fick's second law of diffusion out of a spherical SC depot in Eq. (4), that is,

$$
C(r, t)=C_{0} \sum_{n=1}^{\infty} E_{n} \exp \left(-k_{n}^{2} \frac{D}{R_{d}^{2}} t\right) \frac{R_{d}}{r} \sin \left(k_{n} \frac{r}{R_{d}}\right)
$$

\section{REFERENCES}

1. T. Cook and W. P. Sheridan. Development of GnRH antagonists for prostate cancer: new approaches to treatment. Oncologist 5: 162-168 (2000).

2. K. L. Parker and B. P. Schimmer. Pituitary hormones and their hypothalamic releasing factors. In J. G. Hardman, L. E. Limbird, and A. G. Gilman (eds.), Goodman \& Gilman's The Pharmacological Basis of Therapeutics, McGraw-Hill, London, 2001, pp. 1541-1563.

3. A. J. Tilbrook and I. J. Clarke. Negative feedback regulation of the secretion and actions of gonadotropin-releasing hormone in males. Biol. Reprod. 64:735-742 (2001).

4. D. Klingmuller and H. U. Schweikert. Gonadotropin-releasing hormone: physiological and endocrinological aspects. Recent Results Cancer Res. 124:1-6 (1992).

5. T. Reissmann, A. V. Schally, P. Bouchard, H. Riethmiiller, and J. Engel. The LHRH antagonist cetrorelix: a review. Hum. Reprod. Update 6:322-331 (2000).

6. G. F. Weinbauer and E. Nieschlag. LH-RH antagonists: state of the art and future perspectives. Recent Results Cancer Res. 124: 113-136 (1992).

7. G. Jiang, J. Stalewski, R. Galyean, et al. GnRH antagonists: a new generation of long acting analogs incorporating p-ureido-phenylalanines at positions 5 and 6. J. Med. Chem. 44:453-467 (2001).

8. G. de Pinieux, M. E. Legrier, F. Poirson-Bichat, et al. Clinical and experimental progression of a new model of human prostate cancer and therapeutic approach. Am. J. Pathol. 159:753-764 (2001).

9. P. Broqua, P. J. Riviere, P. M. Conn, J. E. Rivier, M. L. Aubert, and J. L. Junien. Pharmacological profile of a new, potent, and long-acting gonadotropin- releasing hormone antagonist: degarelix. J. Pharmacol. Exp. Ther. 301:95-102 (2002).

10. H. Zhou. Pharmacokinetic strategies in deciphering atypical drug absorption profiles. J. Clin. Pharmacol. 43:211-227 (2003).

11. S. L. Beal and L. B. Sheiner. NONMEM User's Guides. NONMEM Project Group, University of California, San Francisco, 1994.

12. A. Racine-Poon and J. Wakefield. Statistical methods for population pharmacokinetic modeling. Stat. Methods Med. Res. 7:6384 (1998).

13. E. N. Jonsson and M. O. Karlsson. Xpose-an S-PLUS based population pharmacokinetic/pharmacodynamic model building aid for NONMEM. Comput. Methods Programs Biomed. 58:5164 (1999).

14. E. Mosekilde, K. S. Jensen, C. Binder, S. Pramming, and B. Thorsteinsson. Modeling absorption kinetics of subcutaneous injected soluble insulin. J. Pharmacokinet. Biopharm. 17:67-87 (1989).

15. G. Nucci and C. Cobelli. Models of subcutaneous insulin kinetics. A critical review. Comput. Methods Programs Biomed. 62:249257 (2000).

16. P. Wach, Z. Trajanoski, P. Kotanko, and F. Skrabal. Numerical approximation of mathematical model for absorption of subcutaneously injected insulin. Med. Biol. Eng. Comput. 33:18-23 (1995).

17. A. Fick. Über diffusion. Ann.Physik 94:59-86 (1855).

18. C. de Boor. A Practical Guide to Splines, Springer-Verlag, New York, 2001.

19. T. Hastie, R. Tibshirani, and J. Friedman. The Elements of Statistical Learning; Data Mining, Inference, and Prediction, Springer-Verlag, New York, 2001.

20. K. E. Fattinger, L. B. Sheiner, and D. Verotta. A new method to explore the distribution of interindividual random effects in nonlinear mixed effects models. Biometrics 51:1236-1251 (1995). 
21. D. Verotta. Estimation and model selection in constrained deconvolution. Ann. Biomed. Eng. 21:605-620 (1993).

22. S. Winsberg and J. O. Ramsay. Monotonic transformations to additivity using splines. Biometrika 67:669-674 (1980).

23. B. Wang, T. M. Ludden, E. N. Cheung, G. G. Schwab, and L. K. Roskos. Population pharmacokinetic-pharmacodynamic modeling of filgrastim (r-metHuG-CSF) in healthy volunteers. J. Pharmacokinet. Pharmacodyn. 28:321-342 (2001).

24. M. O. Karlsson, E. N. Jonsson, C. G. Wiltse, and J. R. Wade.
Assumption testing in population pharmacokinetic models: illustrated with an analysis of moxonidine data from congestive heart failure patients. J. Pharmacokinet. Biopharm. 26:207-246 (1998)

25. H. Agerso, W. Koechling, M. Knutsson, R. Hjortkjaer, and M. O. Karlsson. The dosing solution influence on the pharmacokinetics of degarelix, a new GnRH antagonist, after s.c. administration to Beagle dogs. Eur. J. Pharm. Sci. 20:335-340 (2003).

26. G. B. Arfken and H. J. Weber. Mathematical Methods for Physicists: International Edition, Academic Press, San Diego, 1995. 